\title{
Evaluation of different heavy-metal stains and embedding media for phase contrast tomography of neuronal tissue
}

Eckermann, Marina, Töpperwien, Mareike, Ruhwedel, Torben, Möbius, Wiebke, Salditt, Tim

Marina Eckermann, Mareike Töpperwien, Torben Ruhwedel, Wiebke Möbius, Tim Salditt, "Evaluation of different heavy-metal stains and embedding media for phase contrast tomography of neuronal tissue," Proc. SPIE 11113, Developments in X-Ray Tomography XII, 111130N (27 September 2019); doi: 10.1117/12.2528432

EDent: SPIE Optical Engineering + Applications, 2019, San Diego, California, United States 


\title{
Evaluation of heavy-metal stains and embedding media for phase-contrast tomography of neuronal tissue
}

\author{
Marina Eckermann ${ }^{a}$, Mareike Töpperwien $^{a}$, Torben Ruhwedel $^{b}$, Wiebke Möbius ${ }^{b}$ and Tim \\ Salditt $^{a}$ \\ ${ }^{a}$ Institute for X-ray Physics, Göttingen University, \\ Friedrich-Hund-Platz 1, 37077 Göttingen, Germany; \\ ${ }^{b}$ Max-Planck-Institute for Experimental Medicine, \\ Hermann-Rein-Straße 3, 37073 Göttingen, Germany
}

\begin{abstract}
In the present work, we evaluate and compare the contrast and resolution obtained on neuronal tissues, i.e. human and mouse cerebellar tissue, with propagation-based $x$-ray phase-contrast computed tomography (PB-CT). At our laboratory-based liquid metal-jet setup, we obtain overview datasets at sub-micron resolution of $\mathrm{mm}^{3}$ sized volumes. In order to evaluate these parameters down to the sub-cellular level, we utilize the synchrotron endstation GINIX at P10, DESY. At this dedicated endstation developed and operated by our group, we utilize $\mathrm{x}$-ray waveguide optics for high-resolution cone-beam scans at strong geometrical magnification. Exploiting this multi-scale approach, we investigate the image quality of cerebellum tissue treated with heavy-metal stainings based on $\mathrm{OsO}_{4}$, PTA, UA, combinations thereof, and metallic mercuric sulfides (Golgi-Cox protocol). In addition, we study the electron density contrast in unstained tissues. Depending on the stain, epoxid resin or paraffin is utilized as embedding media, which also significantly affects contrast and image quality. With this work, we want to contribute to an optimized sample preparation to study the neuronal architecture of the brain tissue in greater detail in three dimensions.
\end{abstract}

Keywords: Propagation-based phase-contrast tomography, heavy-metal staining, contrast-optimized sample preparation, 3d histology of the cerebellum

\section{INTRODUCTION}

Phase-contrast micro- ${ }^{1-3}$ and nanoCT ${ }^{4,5}$ of soft tissues and in particular biopsies and autopsies of the nervous system has been recently demonstrated to reveal cellular and sub-cellular structural details even in unstained tissue samples. ${ }^{5-7}$ However, it is long known that staining and labeling by heavy atoms can significantly increase contrast and signal of small structural features for electron and x-ray microscopy alike. This has been exploited since long for contrast variation in absorption-based radiography, in particular in angiography for visualisation of small capillaries, ${ }^{8}$ contrasted by dissolved $\mathrm{BaSO}_{4}, \mathrm{CaSO}_{4}$, or $\mathrm{SrSO}_{4}$ salts. In the context of PB-CT, suitable stains and/or labels are also highly desirable for increased sensitivity to tissue component, for contrast variation and to obtain specific readouts. For small organisms, iodine and PTA stains are well established, see, ${ }^{9,10}$ but for soft tissue of mammals and in particular of human, suitability of phase-contrast agents is less well explored. Tissue components containing high concentrations of fatty acids can be contrasted by $\mathrm{OsO}_{4},{ }^{11-14}$ based on protocols from EM. This has been exploited for $\mu$ - and nanoCT of myelinated nerve fibers. ${ }^{4,14,15}$ A novel eosin-based staining method for tissue blocks has been presented in. ${ }^{16}$ For neuronal tissue, volume stains for electron microscopy (EM) have recently gained increased interest in view of connectomics, ${ }^{17,18}$ notably $\mathrm{rOTO} .{ }^{19-21}$

Generally, we are interested in the suitability of heavy-metal stains for tissue of the nervous system which can augment the capabilities of propagation-based x-ray phase-contrast tomography of neuronal tissue. For this investigation, we chose tissue from cerebellum, since it has a well-described morphology and cyto-architecture. Furthermore, unstained tissue of human cerebellum has previously been studied by absorption- ${ }^{22}$ and phasecontrast CT enabling detection of single Purkinje cells ${ }^{23-25}$ and subsequently also smaller cells such as granules, ${ }^{7}$

Further author information: (Send correspondence to Tim Salditt)

Tim Salditt: E-mail: tsaldit@gwdg.de, Telephone: +495513925556

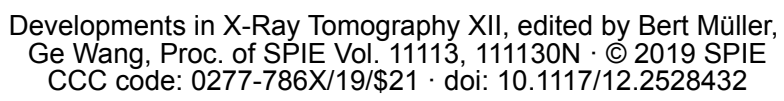

Proc. of SPIE Vol. $11113111130 \mathrm{~N}-1$ 
showing impressive level of detail.

Analogous to comparable high-resolution imaging methods such as scanning electron microscopy (SEM) or light microscopy-based histology, the effect from staining on contrast is studied here. To this end, modified versions of the $r O T O$-protocol ${ }^{26}$ are used, as detailed below.

The $r O T O$ is a standard, $\mathrm{OsO}_{4}$-based staining procedure in three-dimensional (3d) SEM, a technique that requires enhanced heavy-metal impregnation for contrast formation using backscatter electron detection. It stands for "reduced osmium, thiocarbohydrazide (TCH), osmium". The contrast-enhancing properties of osmium tetroxide $\mathrm{OsO}_{4}$ (atomic weight: 190.2) are mainly due to aggregation of lower oxides of Os, and originate only to a minor degree from unreduced Os. ${ }^{27}$ It binds to the phospholipid headgroups, hence contrasting plasma membranes, multi-vesicular structures, ribosomes and Golgi complexes. We hence expect in particular higher contrast for membrane-rich structures in the cerebellum such as fiber tracts. Not withstanding contrast enhancement, $\mathrm{OsO}_{4}$ 's main purpose in EM is serving as a chemical fixative, enabling structure preservation by inactivation of osmolarity. ${ }^{28}$ In $\mathrm{rOTO}$, TCH is applied in between two consecutive $\mathrm{OsO}_{4}$ immersions. TCH highlights carbohydrates in SEM or nerve tissue in light microscopy, ${ }^{27}$ but its main purpose here is to increase $\mathrm{OsO}_{4}$ 's membrane penetration.

Another standard substance to increase feature contrast in $\mathrm{OsO}_{4}$-fixated tissue in SEM is phosphotungstic acid $\left(\mathrm{H}_{3} \mathrm{PO}_{4} \cdot 12 \mathrm{WO}_{3} \cdot 24 \mathrm{H}_{2} \mathrm{O}\right.$, PTA, atomic weight: 183.92). ${ }^{27}$ This contrast agent functions via binding to hydroxyl groups due to its anionic nature. It selectively decolors compounds by exchanging their anions, and its performance is directly connected to the buffers' $\mathrm{pH}$ level: ${ }^{27}$ at $\mathrm{pH} \leq 2.0$, it is sensitive for polysaccharides with a feature specificity depending on the exact $\mathrm{pH}$ value, while $\mathrm{pH} \approx 3.0-3.5$ turned out advantageous for nucleoproteins, and $\mathrm{pH} \approx 4.0$ for glycogen and nucleoproteins. In general, for $\mathrm{pH} \geq 7.3$, PTA stains inefficiently. Due to the requirement of low $\mathrm{pH}$ levels, PTA treatments usually demand adequate pre-fixation for tissue protection. Also, PTA itself may serve tissue fixation. Applied to brain tissue, PTA especially binds to synaptic complexes, fibres and membranous structures. ${ }^{27}$

Uranyl acetate $\left(\mathrm{UO}_{2}\left(\mathrm{CH}_{3} \mathrm{COO}\right)_{2} \cdot 2 \mathrm{H}_{2} \mathrm{O}\right.$, UA), with an atomic weight of 238.07 , is one of the heaviest stain used in EM. ${ }^{27}$ Also for this stain, a low $\mathrm{pH}$ level is crucial, ideally $\sim 1.0-2.0$. It binds particularly well to nucleic acids and phosphate groups, and less so to proteins depending on their charge, hence highlighting for example nucleo-histones in DNA or membranous structures. Also, when combined with reduced Os from (brain) tissue pre-treatment, UA may function as a morphological stabilizer. Like $\mathrm{OsO}_{4}$ and PTA, UA serves both staining and fixation. All three substances perform effectively when combined with prior glutaraldehyde treatment. ${ }^{27}$

The primary goal of this work is to test different $r O T O$-derived heavy stains for their suitability in propagationbased x-ray phase-contrast tomography. Apart from these stains developed in the field of EM, we also include one of the oldest heavy atom stains of histology, notably the Golgi-Cox stain, which has already proven suitable for phase-contrast x-ray tomography. ${ }^{29,30}$ As reviewed in ${ }^{31}$ it is a derivative of Camillo Golgi's method, who discovered the penetration of metals in neurons in $1873 .{ }^{32-34}$ S.R. Cajal's works are well-known for early images and studies of glial and dendritic architecture using Golgi's protocol. ${ }^{35}$ The key of the protocol's success is its sparse staining of only $\sim 3-10 \%$ of all neuronal features in a structure-rich tissue. The Golgi protocol consists of sample treatment with a potassium chromate and potassium dichromate mixture, followed by silver nitrate. A further development has been made by Cox, who established addition of mercurid chloride to the potassium chromate and potassium dichromate mixture, changing its $\mathrm{pH}$-level for a better tissue impregnation. This modification is referred to as Golgi-Cox method: In a first step, tissue forms compounds with mercuric chloride, which are then transformed to metallic mercuric sulfides (molar weight: $232.66 \mathrm{~g} / \mathrm{mol}^{36}$ ) in a later step. It is considered to be a structure-preserving staining technique for axons and dendrites.

In this work, we combine laboratory micro-PB-CT (lab- $\mu \mathrm{CT})^{37,38}$ and high-resolution synchrotron radiation computed tomography (SR-CT) based on a focusing unit composed of Kirkpatrick-Baez mirrors and an x-ray waveguide. ${ }^{39}$ For notational clarity, we next briefly introduce phase-contrast x-ray imaging by propagation, and the basics of phase retrieval as used here.

Phase contrast is governed by the real part decrement $\delta(\vec{r}, E)$ of the x-ray refractive index $n(\vec{r}, E)=1-$ $\delta(\vec{r}, E)+i \beta(\vec{r}, E)$. The imaging regime is governed by the Fresnel number $F$. The lab- $\mu \mathrm{CT}$ data is recorded in the direct contrast or edge enhancement regime, defined by

$$
F=\frac{p x_{\mathrm{eff}}^{2}}{z_{\mathrm{eff}} \lambda}=\frac{p x^{2}}{z_{12} M \lambda} \approx 1
$$


where $p x_{\text {eff }}=p x / M$ for the effective pixel size and $z_{\text {eff }}=z_{12} / M$ for the effective propagation distance have been used, based on the respective real values $p x$ and $z_{12}$, exploiting the Fresnel scaling theorem at geometrical magnification $M .{ }^{40}$ In this regime, a reconstruction scheme known as Bronnikov-aided correction (BAC) ${ }^{41}$ can be used, which is compatible low coherence sources and which preserves image sharpness. ${ }^{37,38}$ BAC can be derived from the transport of intensity equation (TIE), which describes the propagation of a paraxial wave along $\vec{z}$ with intensity $I(\vec{r})$ and phase distribution $\phi(\vec{r}): \nabla_{\perp}\left(I(\vec{r}) \cdot \nabla_{\perp} \phi(\vec{r})\right)=-k \partial_{z} I(\vec{r}), k$ being the wavenumber. For small propagation distances $z_{\text {eff }}$ and a purely phase shifting object, the TIE can be linearized, and an approximated phase $\tilde{\phi}$ can be computed $\operatorname{as}^{42,43}$

$$
\tilde{\phi}\left(\vec{r}_{\perp}\right)=2 \pi F \cdot \mathcal{F}_{\perp}^{-1}\left[\frac{\mathcal{F}_{\perp}\left[\frac{I\left(\vec{r}_{\perp}, z\right)}{I_{0}}-1\right]}{|\vec{k} \perp|^{2}+\alpha}\right],
$$

where $\mathcal{F}$ denotes the Fourier transform and $I_{0}$ the (uniform) intensity distribution. The parameter $\alpha$ serves to regularize the singularity at zero spatial frequency and consider residual absorption in the sample, and in practice is chosen such that edge enhancement is canceled. In a second step, the approximated phase $\tilde{\phi}$ is used to compute a corrected, sharp intensity distribution in the object exit plane, according to ${ }^{41}$

$$
I\left(\vec{r}_{\perp}, z=0\right)=\frac{I\left(\vec{r}_{\perp}, z\right)}{1-\gamma \nabla_{\perp}^{2} \tilde{\phi}\left(\vec{r}_{\perp}\right)} .
$$

The two regularization parameters $\alpha$ and $\gamma$ are chosen such that the resulting 'hypothetical' intensity distribution contains contributions from intensity and phase contrast.

The SR-CT data is recorded in the holographic regime at $F \ll 1$, offering higher contrast for high spatial frequencies compared to the direct-contrast regime. For weakly absorbing and homogeneous objects, the contrasttransfer function $(\mathrm{CTF})$ is given by ${ }^{44-46}$

$$
\frac{I\left(\vec{k}_{\perp}, z\right)}{I_{0}}=2 \pi \delta_{D}\left(\vec{k}_{\perp}\right)+2 \sin (\chi) \cdot \phi\left(\vec{k}_{\perp}\right)-2 \cos (\chi) \cdot \xi\left(\vec{k}_{\perp}\right),
$$

with $\chi=\frac{\lambda z k_{x}^{2}}{4 \pi}$, the Dirac-delta function $\delta_{D}\left(\vec{k}_{\perp}\right)$ and the absorption coefficient $\xi\left(\vec{k}_{\perp}\right)$. From the CTF, an expression for the phase in the object plane can be derived: ${ }^{46}$

$$
\phi\left(\vec{r}_{\perp}\right)=\mathcal{F}_{\perp}^{-1}\left[\frac{\sum_{d=1}^{N}\left(\sin \left(\chi_{d}\right)+\frac{\delta}{\beta} \cos \left(\chi_{d}\right)\right) \cdot \mathcal{F}_{\perp}\left[I\left(\vec{r}_{\perp}, z_{d}\right)-1\right]}{\sum_{d=1}^{N} 2\left(\sin \left(\chi_{d}\right)+\frac{\delta}{\beta} \cos \left(\chi_{d}\right)\right)^{2}+\alpha}\right],
$$

where $\frac{\delta}{\beta}$ is assumed to be constant (homogeneous object approximation), and $\alpha$ is a regularization parameter. $N$ is the number of exposures recorded at different defocus distances (Fresnel numbers) in order to sample all spatial frequencies at high contrast. For the exact implementation of the regularization scheme used here, see also. ${ }^{47}$

\section{METHODS}

\subsection{Experimental setups}

\subsubsection{Laboratory-based setup}

Fig. 1a) depicts the geometry of the home-built laboratory microCT setup (lab- $\mu \mathrm{CT}$ ) operated at a liquid metal jet x-ray source (Excillum, Stockholm, Sweden). ${ }^{15,48}$ The anode material Galinstan (68.5\% Ga, 21.5\% In, $10 \%$ $\mathrm{Sn}$ ) is liquid at room temperature, allowing for higher thermal load and hence a corresponding brilliance gain beneficial for phase contrast. ${ }^{49}$ Phase-contrast tomography using this source has been presented in. ${ }^{50-52}$ The source was operated with source size $10 \mu \mathrm{m}, \mathrm{e}^{-}$-beam current $1.4 \mathrm{~mA}$, and $40 \mathrm{kV}$ voltage, resulting in corresponding cutoff photon energy of the bremsstrahlung. The dominant energy for phase contrast is given by the characteristic line $K_{\alpha, \mathrm{Ga}}=9.25 \mathrm{keV}$. The sample stage positioned at a distance $z_{01}$ from the source is equipped with 6 motors 


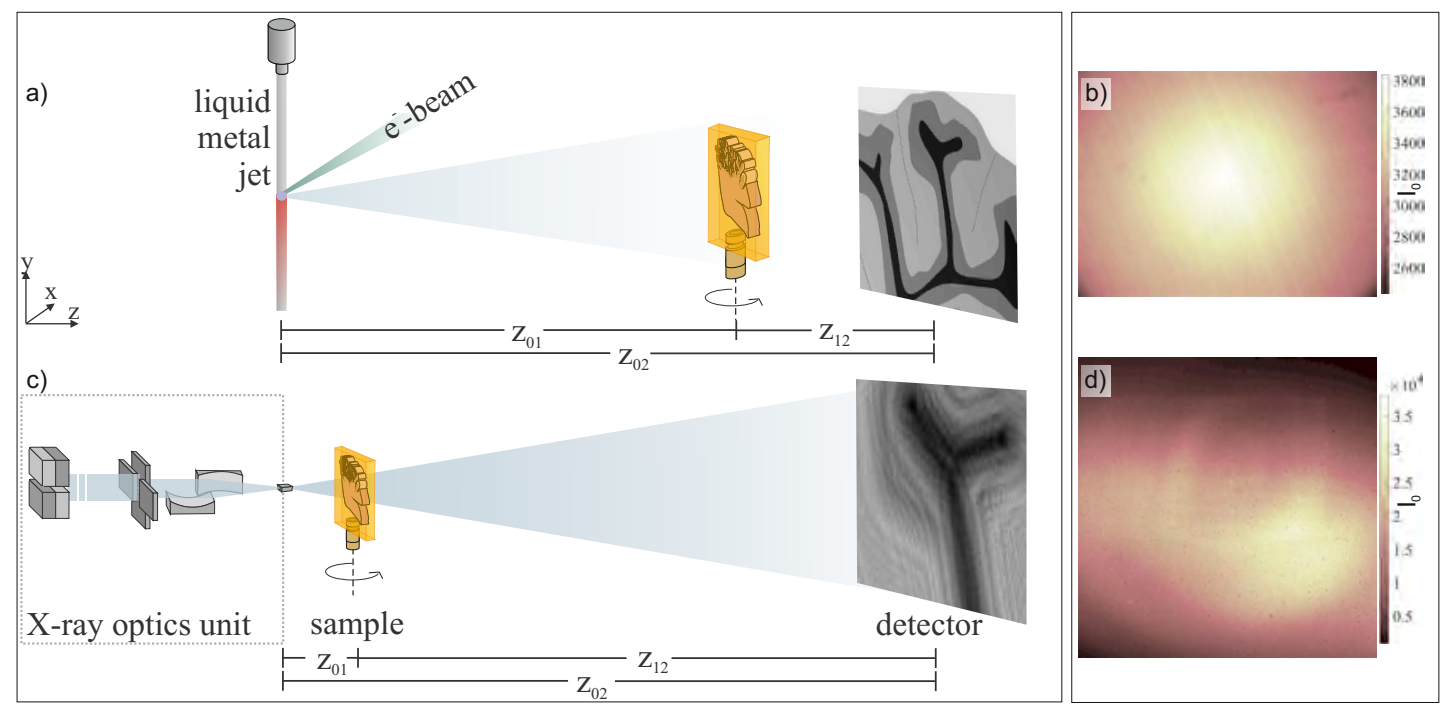

Figure 1. Illustrations of the experimental setups: (a) Lab- $\mu$-CT setup, with liquid metal jet anode, high lens-coupled scintillator detector and magnification $M \simeq 1$, i.e. $z_{02} \ll z_{01}$. (c) SR-CT setup with KB/waveguide optics. KB-mirrors focus the monochromatized undulator beam onto the waveguide. The waveguide exit beam is used for holographic illumination at high $M$. (b \& d) Examples of flat-field images for the two setups. Due to coherent multi-modal superposition, the waveguide flat-field shows modulations.

(1 rotation, 5 translations), allowing for alignment of the rotation axis and the sample. The detector is positioned along a rail at distance $z_{02}$, and can be translated on top along $x$ and $y$. Adjustment of system resolution as a function of spot size, detector pixel size and geometry $\left(z_{01}, z_{02}\right)$ for the setup has been discussed in. ${ }^{37,48,53}$ For most scans presented here, we have used the lens-coupled single-crystal scintillator-based CCD-detector Xsight (Rigaku, Tokyo, Japan) with $2504 \times 3326$ pixels $(\mathrm{h} \times \mathrm{w})$ with a $10 x$-objective, resulting in $p x=0.54 \mu m$. To reach a resolution below source size based on the high resolution capability of the detector, small geometrical magnification $M=\frac{z_{02}}{z_{01}}=\frac{176.6 \mathrm{~mm}}{155.0 \mathrm{~mm}} \approx 1$ was chosen. ${ }^{37}$ Empty beam corrected data were corrected for hot pixels. To increase the signal-to-noise-ratio (SNR), pixels were $2 \times 2$-binned. The acquisition parameters resulted in $F=0.35$, corresponding to the direct contrast regime. Fig. 1b) gives a flat-field image of the setup, showing the influence of the objective on the illumination profile. X-ray phase-contrast tomography scans were recorded with 1001 angular positions at an acquisition time of 50s. For the overview scan of the Golgi-Cox stained mouse brain, a CMOS flat panel detector (Dexela, PerkinElmer, Waltham MA, USA) with $1536 \times 1944$ pixels $(\mathrm{h} \times \mathrm{w})$ and $p x=75 \mu \mathrm{m}$ was used. Its rather large sensitive area is advantageous for large FOV at moderate geometrical magnification, $M=\frac{z_{02}}{z_{01}}=\frac{1739.2 \mathrm{~mm}}{127.5 \mathrm{~mm}} \approx 14$ namely, providing $p x_{\mathrm{eff}}=5.5 \mu \mathrm{m}$ and $F=1.91$. As shown before, phase reconstruction with BAC (Eq. 3) gives excellent results for 3d histology.3,15,37 Prior to tomographic reconstruction based on, ${ }^{54,55}$ ring artifacts in the sinograms were mitigated. ${ }^{56}$

\subsubsection{Synchrotron radiation-based setup}

After a first screening and overview scans with lab- $\mu \mathrm{CT}$, high resolution scans were performed at the GINIX nanofocus endstation ${ }^{39}$ of the P10 beamline at the PETRAIII storage ring (DESY, Hamburg). As sketched in Fig. 1c), the undulator radiation is monochromatized by a double-crystal $\mathrm{Si}(111)$ monochromator to a photon energy $E=7.9 \mathrm{keV}$, and focused by Kirkpatrick-Baez (KB) mirrors to about $400 x 400 \mu \mathrm{m}^{2}$ (FWHM). The source spot size $s$ relevant for holography was determined by $x$-ray waveguides positioned in the KB-focal plane. Apart from the reduction of $s$ (and correspondingly increase of numerical aperture), waveguide optics increases spatial coherence by mode filtering ${ }^{57}$ and delivers smooth illumination wavefronts for holography. ${ }^{58-60}$ For this experiment, air-filled channel waveguides in silicon or germanium were used, fabricated by e-beam lithography, reactive ion etching and wafer bonding. ${ }^{61}$ Channels with different lateral size and depth were used, in the order 


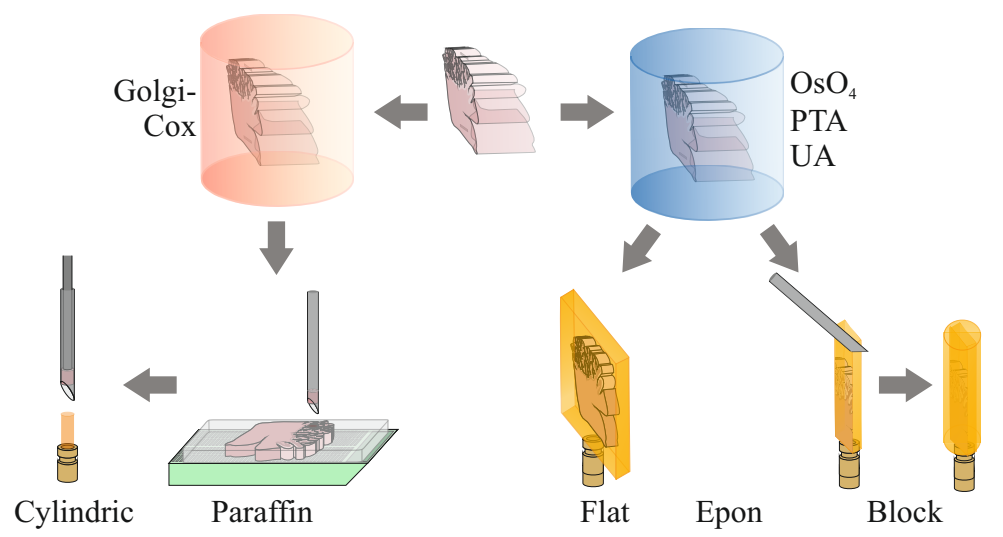

Figure 2. Overview on the sample preparations involving (left) Golgi-Cox and (right) the rOTO protocol (with modifications), based on $\mathrm{OsO}_{4}, \mathrm{PTA}$ and UA.

of $10^{2} \mathrm{~nm}$. Note that the actual beam confinement is given by the width of the confined beam intensity, which is smaller than the dimensions of the rectangular channel cross section. This value for $s$ can be retrieved by inversion of the far-field pattern. ${ }^{58,59}$ Scans were recorded at magnification $M=\frac{z_{02}}{z_{01}}=\frac{5126 \mathrm{~mm}}{138 \mathrm{~mm}} \approx 37 \gg 1$. A sCMOS camera with Gadox scintillator and $2048 \times 2048$ pixels with $p x=6.5 \mu m$ (Photonic Science, UK) was used, resulting in $p x_{\text {eff }}=176 \mathrm{~nm}$. Accordingly, data were recorded in the holographic regime with $F=0.0015$, and phase reconstruction was performed by CTF (eq. 5). An exemplary flat-field image is given in Fig. 1d). Due to changes in the electron beam orbit, as well as drift in the beamline optical components (in particular due to cooling of the monochromator), the waveguide intensity and mode distribution changed, and frequent realignments were required. The acquisition time was $\sim 1 s$ per projection. In order to achieve larger penetration depth, the setup was also adjusted for $13.8 \mathrm{keV}$ x-rays.

GINIX also allows for parallel beam tomography, which can be implemented in parallel to cone beam after slight rearrangement of optics, and used on the same sample, as we will report in detail elsewhere. This parallelbeam data (presented in Fig. 8) was recorded at $M=\frac{z_{02}}{z_{01}}=\frac{150 \mathrm{~mm}}{138 \mathrm{~mm}} \approx 1$ and $F=0.0121$, and reconstructed by BAC. For data from both configurations, empty-beam-corrected images were filtered for hot pixels, and after phase reconstruction filtered for ring artifact mitigation using. ${ }^{56,62}$ If required, sinograms were further smoothed in prior to the tomographic reconstruction, which was based on the Matlab (The MathWorks, Inc., MA, USA)-integrated function iradon with a standard Ram-Lak filter.

\subsection{Sample preparation}

\subsection{1 rOTO-based staining}

As rOTO is an established protocol for large volume imaging by SEM, a method which requires enhanced contrast, its suitability for X-ray phase-contrast tomography has to be tested. Cerebellar tissue from the same adult C57/Bl6 wild type mouse was used for all samples with this stain. Animals were deeply anesthesized and perfused with Karlsson-Schultz (K\&S) solution, consisting of $2.5 \%$ glutaraldehyde and $4 \%$ formaldehye in phosphate buffer, following the procedures as described in. ${ }^{63}$ The cerebellum was dissected and chemically postfixated for $24 \mathrm{~h}$. The tissue block was then cut into 200-500 $\mu \mathrm{m}$ sagital slices with a vibratome. In between each step, samples were washed for $15 \mathrm{~min}$. Before staining, the tissue was first placed into $0.1 \mathrm{~mol}$ phosphate buffer for $15 \mathrm{~min}$. First incubation was carried out with reduced osmium (i.e. $\mathrm{OsO}_{4}$ with added potassium ferrocyanide) for either 30 or $60 \mathrm{~min}$ at room temperature. As $\mathrm{rOTO}^{26}$ stands for "reduced osmium, TCH, osmium", tissue was then placed into the osmiophilic TCH solution for $1 \mathrm{~h}$, followed by again 30 or $60 \mathrm{~min}$ reduced osmium. $\mathrm{OsO}_{4}$ does not only stain, but also chemically stabilizes the molecular tissue structure. For some tissue pieces, the protocol was continued with a $15 \mathrm{~min}$ incubation step with $2 \% \mathrm{UA}$ or $2 \% \mathrm{PTA}$ at $4^{\circ} \mathrm{C}$. To investigate the staining properties of solely UA or PTA, the rOTO protocol was modified: the $\mathrm{OsO}_{4}$ steps were skipped for some samples. However, the fixation effect of $\mathrm{OsO}_{4}$ 's is crucial for brain tissue, such that three of these samples desintegrated during preparation, and only one sample stained with UA for 30min remained. Via increasing 
aceton and then epoxid-resin-in-aceton series, tissue was dehydrated and fully penetrated by the resin. When kept at $60^{\circ} \mathrm{C}$ for $24 \mathrm{~h}$, the epoxid resin polymerizes. As indicated on the right of Fig. 2, samples were either prepared to be "flat" (0.8-2 $\mathrm{mm}$ laterally, 30-60 $\mathrm{m}$ in depth) or trimmed with the microtome by using a $90^{\circ}$ diamond knife to form "blocks" (360-530 $\mu \mathrm{m}$ laterally, 170-500 $\mu \mathrm{m}$ in depth). For the latter, sample surfaces were smoothened by applying another thin layer of epoxid resin.

\subsubsection{Golgi-Cox-based staining}

Apart form rOTO, which was the main focus in this work, we also used Golgi-Cox staining. The Golgi-Cox method was developed to give specific contrast to neurons, including their dendrites and spines. For this purpose, we used the kit provided by FD NeuroTechnologies' (Columbia MD, USA) FD Rapid GolgiStain Kit, which is based on the protocol by Ramón-Moliner ${ }^{64}$ and Glaser and Van der Loos. ${ }^{65}$ 24h prior to the start of the preparation, solution $A$ and $B$ are mixed 1:1. They involve mercury, potassium chromate and potassium dichromate, a highly toxic mixture which may be fatal in case of misuse. The top part of the mixture is sucked off and used as stain, avoiding the solid particles which settle at the bottom. As this staining procedure also includes the required chemical fixation, the cerebellum tissue was freshly removed from the animal, carefully rinsed with ultrapure water (Milli-Q), and placed into the mixture, and subsequently always stored in the dark. For one mouse brain, $\sim 10 \mathrm{ml}$ was used and replaced after $6 h$ or the next day. After 14 days at room temperature, the tissue was transferred to solution $C$ for $72 h$ at room temperature, and renewed the next day. Then, tissue blocks were washed with Milli-Q twice for 10-20min. Then, sections were put into a 1:1:2 mixture of solution $D, E$ and Milli-Q for $\sim 30 \mathrm{~min}$. This is a crucial step, since it serves for the metal-formation of the stain (cf. section 1). Again, the tissue is washed twice with Milli-Q for 10-20 min. The stained tissue blocks were then paraffinembedded, involving an ethanol-based dehydration series, washing with xylene and full tissue penetration series with paraffin at $60^{\circ} \mathrm{C}$, turning solid at room temperature. As shown in Fig. 2, the stained cerebellum blocks were mounted on paraffin-embedding cassettes. Using a $1 \mathrm{~mm}$ biopsy punch, smaller cylindrical samples were extracted and inserted into Kapton polyimide tubes (Professional Plastics, Fullerton CA, USA) for high-resolution scans.

\subsubsection{Preparation of control samples}

In order to clearly evaluate whether observations are made based on the respective heavy-metal stain, on the tissues itself or the experimental setup, unstained control samples were included: Firstly from a previous study, ${ }^{7}$ an unstained human cerebellum autopsy, 10\% PFA-fixated and paraffin-embedded, provided by C. Stadelmann (Institute for Neuropathology, UMG, Göttingen). In addition, a K\&S-fixated and paraffin-embedded mouse cerebellum was included. Autopsy samples were extracted using a $1 \mathrm{~mm}$ biopsy punch and inserted into polyimide tubes.

\section{RESULTS}

Fig. 3 presents lab- $\mu \mathrm{CT}$ results for the unstained controls of cerebellum tissue (mouse and human). (a) illustrates its basic anatomy with a more detailed zoom-in view. The white matter (WM) contains myelinated neuronal structures such as axons, forming fiber tracts (FT). Fibers originating in the WM traverse the granular layer (GL) unmyelinated, consisting of granular cells (GC) and Golgi cells (GgC). A single layer of Purkinje cells (PC, PCL) is located in direct proximity of the GL, with sparse occurrences of basket cells (BC). PCs' long and widely branched dendritic trees penetrate into the molecular layer (ML), which distinguishes itself from the GL by its larger and more sparsely spreaded cells, the molecular cells (MC). The external granular layer (EGL) borders the ML. The EGL is a characteristic structure for brain development.

\section{1 rOTO-based \& control preparations}

Prior to valuable synchrotron beam time, samples were screened with the Xsight-based lab- $\mu \mathrm{CT}$ presented in section 2.1.1. Fig. 3 presents virtual slices through the reconstructed $3 \mathrm{~d}$ volumes of the unstained and paraffinembedded autopsies. For phase-retrieval, (human) $\alpha_{\mathrm{BAC}}=0.05$ and $\gamma_{\mathrm{BAC}}=0.17$ and (mouse) $\alpha_{\mathrm{BAC}}=0.008$ and $\gamma_{\mathrm{BAC}}=0.16$ were chosen based on visual inspection. For both preparations, the characteristic tissue regions (WM, GL, PCL and ML) with their corresponding cells (GC, PC and MC) are clearly recognized. However, in particular for $\mathrm{PCs}$, there appears to be a feature contrast gradient within the mouse tissue, indicating fixation 

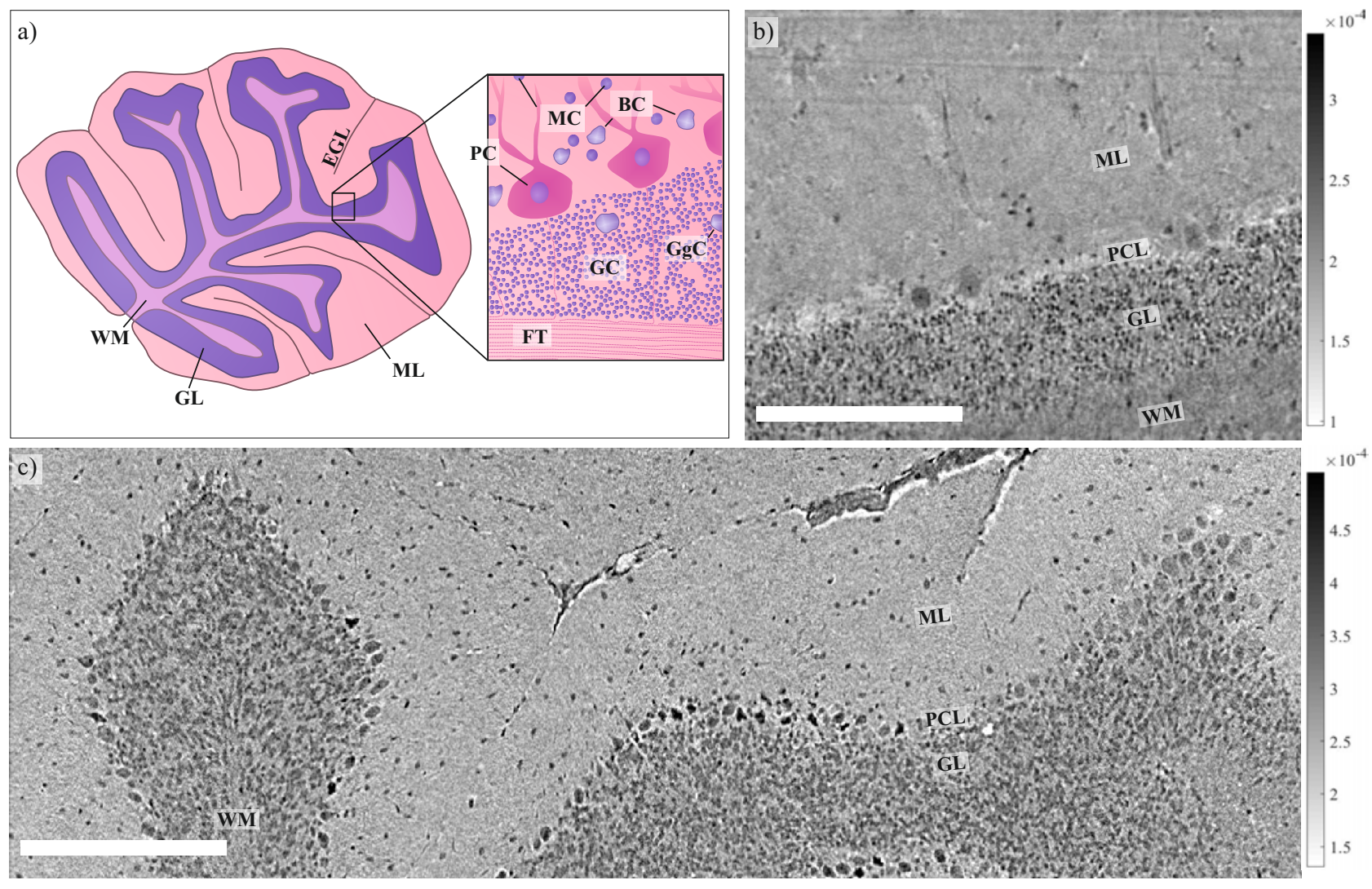

Figure 3. (a) Schematic drawing of the cellular anatomy of the cerebellum.

Overview on data achieved from unstained, paraffin-embedded cerebellum tissues with the lab- $\mu$ CT setup, presented as effective absorption coefficient per voxel. Virtual slices in the yz-plane through data from (b) human and (c) mouse tissue. Scale bars: $200 \mu m$.

inhomogeneities. Note the difference in size of the tissue components between human and mouse, in particular the PCs, which also exhibit different shapes and contrast.

Fig. 4 gives an overview of typical contrast properties of the different rOTO-derived stains, as they appear in lab- $\mu$ CT. Preparations involving $\mathrm{OsO}_{4}$ show particularly high contrast in the WM. Single cellular features emerge to much lower extent than in the data in Fig. 3. Only for $\mathrm{OsO}_{4}$ without additional stain (PTA, UA), some granularity in the GL is visible. Also, the PCL appears to be stained negatively by $\mathrm{OsO}_{4}$. As described in section 1, this may be attributed to the lower density of ribosomes in PCs compared to GC, for instance. When combining $\mathrm{OsO}_{4}$ with PTA or UA, GL and ML also differ in contrast, however without a clear distinction of the PCL. A special case of contrast is noticed for UA-only staining: there is no dominant appearance for the WM, which becomes indistinguishable from the GL. Some granularity is recognized for the ML in this case. The most striking feature is the high contrast of the EGL. As motivated earlier, this structure is predominantly expressed during brain development. From the characteristics of UA binding to membranous and DNA-structures (cf. 1), particular staining behavior of structures at maturation stages becomes conclusive. Given the fact that all the rOTO-derived preparations originate from the very same animal, this structure has not been observed for any of the other staining preparations, in particular not for the combination of $\mathrm{OsO}_{4}$ with UA. However, due to the brittle architecture of cerebellum when not $\mathrm{OsO}_{4}$-treated, this observation is rather wary.

Next, samples were imaged at sub- $\mu \mathrm{m}$ resolution using the SR-CT setup introduced in 2.1.2. The $\delta / \beta$ ratios in CTF-reconstruction were chosen based on physical properties and fine-tuned from visual inspection. Fig. 5 shows the unstained controls, and Tab. 1 the corresponding quality measures. The evaluation of the resolution based on the Fourier-Shell-Correlation (FSC), which is also affected by the overall noise, gives a value 

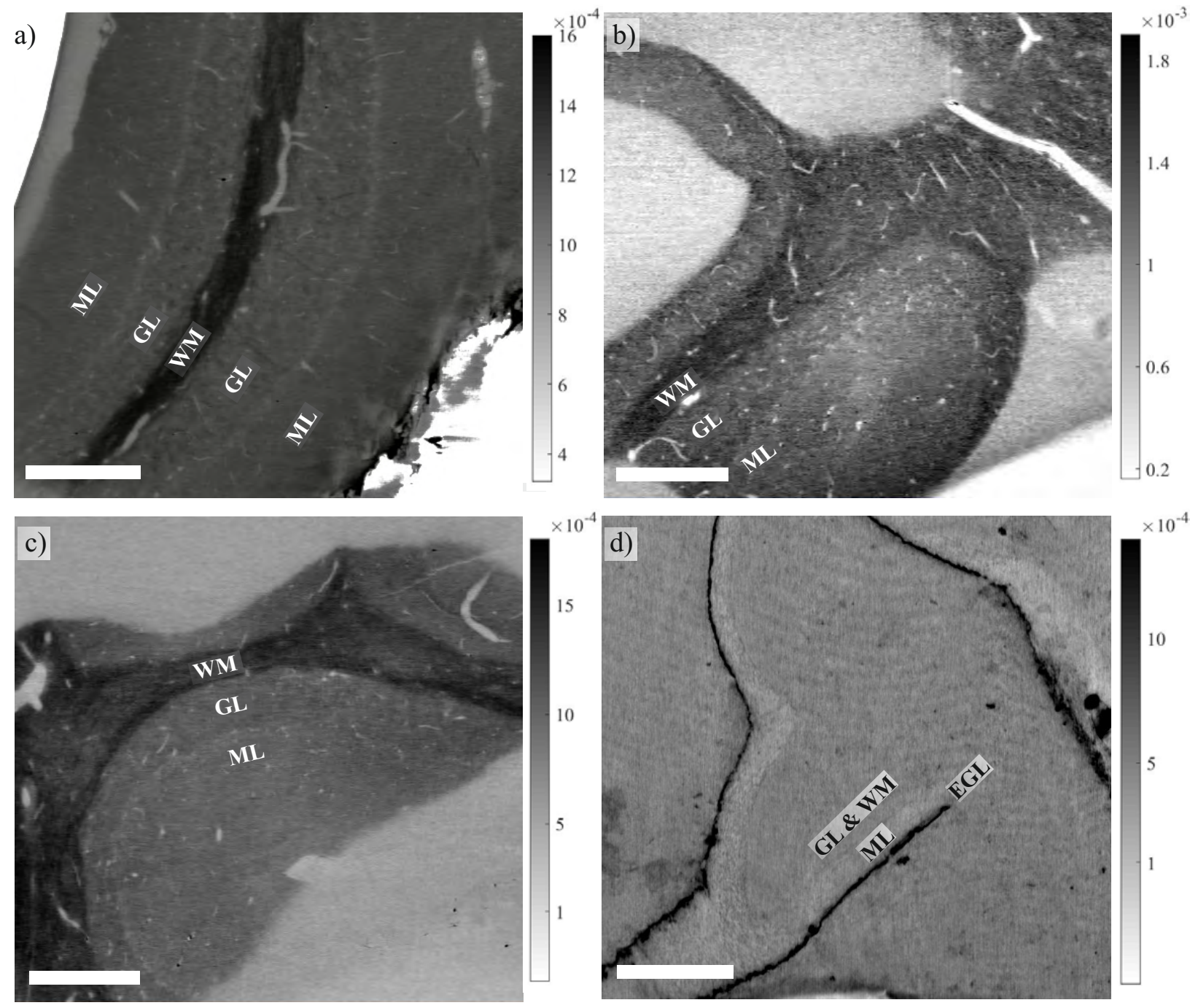

Figure 4. Overview of contrast obtained with different stains (mouse cerebellum) at the lab- $\mu$ CT setup, given as effective absorption coefficient per voxel. The tissue was stained with (a) $\mathrm{OsO}_{4}(30 \mathrm{~min})$, (b) $\mathrm{OsO}_{4} \& \mathrm{PTA}(30 \mathrm{~min})$, (c) OsO 4 \& UA (60min) and (d) UA, and epon-embedded in "flat" geometry. Tab. 2 gives further parameters of preparation and data quality. Scale bars: $200 \mu m$. 

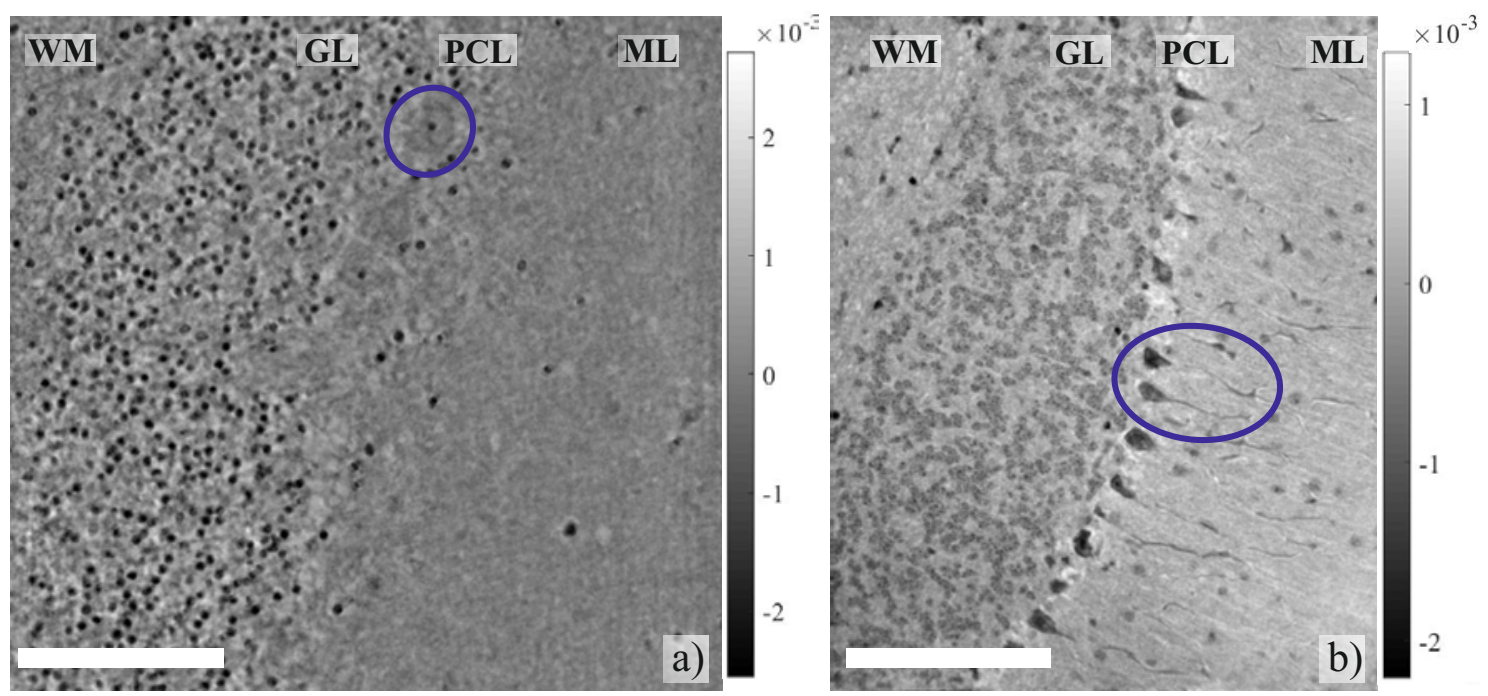

Figure 5. Overview of SR-CT results in terms of phase shift per voxel for the unstained, paraffin-embedded samples, shown for (a) human and (b) mouse cerebellum. Circles mark PCs. Quantitative quality measures are presented in Tab. 1. Scale bars: $90 \mu m$.

of $0.7 \mu \mathrm{m}$, steepness-of-edge at a blood vessel profile about $0.5 \mu \mathrm{m}$. The SNRs of different features in the unstained cerebellum are also given. Note that the background signal for PC nucleus was chosen to be outside-the-PC, not inside-the-PC's body. Visual and quantitative assessment of the PCs' contrast confirm the observations made for the lab- $\mu \mathrm{CT}$ data (Fig. 3). Especially the SNR for the mouse PC body data stands out. The appearance of the PCs' dendritic trees for the mouse tissue sample is particularly remarkable, and may eventually be explained by the superior structure preserving property of K\&S-fixation. Nevertheless, their nuclei turn out fuzzier. While the $\mathrm{GC}$ emerge stronger contrasted for mouse tissue with lab- $\mu \mathrm{CT}$, same is true for the GC in human cerebellum with the synchrotron setup. For both data sets, also blood vessels appear comparably strong due to blood remains.

Fig. 6 presents the results for $r O T O$-preparations, recorded with the SR-CT setup. Directly visible (already without zoom-in) are the blood vessels which appear in negative contrast, since they were emptied by perfusion of the animals before staining. In the case of unstained and paraffin-embedded samples, identification and tracking of blood vessels is also possible, but since tissues were not perfused but chemically fixated by diffusion only, blood remains would complicate the automatic segmentation. Also, the overall contrast between empty vessels and stained tissue is of course huge for all of the stained samples. Next we note, there is no strong overall contrast of the WM, as in the respective lab- $\mu \mathrm{CT}$ data, but the discrimination of the different layers and features emerges on a cellular level. $\mathrm{MC}$ bodies and nuclei, for example, are contrasted in the $\mathrm{OsO}_{4} \& \mathrm{PTA}$ data. As expected, the $\mathrm{OsO}_{4}$-preparations reveal particular contrast for myelineated axons in the FT. Still, SNR-values are lower than for the data from unstained and paraffin-embedded mouse tissue. Same is true for PCs' nuclei. Compared to the other rOTO-derived preparations, PC bodies appear slightly stronger contrasted when PTA is involved. Concerning resolution, steepness-of-edge values are at about the same level for all synchrotron data sets. Contrarily, differences are observed for the more overall-preparation-mirroring FSC-values. For all "flat" preparations, the FSC-resolution is $\approx 2 \mu \mathrm{m}$ and far below capabilities of the instrument, the stains and the tissue. From comparison with the steepness-of-edge, an increased noise level seems to affect the data quality. This can be attributed to artifacts related to facets and in part also to higher absorption for some angles. The "block" geometry, however, does not suffer from these artifacts and approaches the results of the unstained preparations. When $\mathrm{OsO}_{4}$ is combined with PTA or UA, resolution is slightly better than for the unstained samples $(0.55 \mu m$ and $0.56 \mu \mathrm{m}$ vs. $\sim 0.65 \mu \mathrm{m})$. For the combination of $\mathrm{OsO}_{4}$ with PTA, MC bodies and nuclei are recognized as for no other preparation discussed here.

Since the 60 min- $\mathrm{OsO}_{4}$ "flat" preparation of the $\mathrm{OsO}_{4}$ \& PTA-stained tissue resulted in comparably strong 
Table 1. SR-CT data quality metrics for unstained, paraffin-embedded samples. FSC is based on volumes of $200 \times 200 \times 200$ voxels except for ${ }^{+}(150 \times 150 \times 150)$, and computed with with a Kaiser-Bessel window of 7 pixels. ${ }^{66}$ Steepness-of-edge has been evaluated for blood vessels. The SNR was calculated as $\left(\mu_{\mathrm{ft}}-\mu_{\mathrm{bg}}\right) / \sigma_{\mathrm{bg}}$ on neuronal fibers in white matter, Purkinje cells' body and nucleus and blood vessels, respectively. Annotations on SNR marked with * give the number of features evaluated. Empty beam intensities are given in analog-digital-units (ADU). In the case of the human tissue, the position of the WM region was located too close to the edge of the scan volume to perform reasonable evaluation of the axons' contrast, hence "n.a." (not applicable).

\begin{tabular}{|l|cc|}
\hline & \multicolumn{2}{|c|}{ Unstained cerebellum } \\
& human & mouse \\
\hline$\delta / \beta(\mathrm{CTF})$ & 35 & 55 \\
\hline Resolution & & \\
FSC $1 / 2$ bit $(\mu \mathrm{m})$ & 0.69 & 0.65 \\
Steepness-of-edge $(\mu \mathrm{m})$ & 0.44 & 0.53 \\
\hline SNR & & \\
Axon & n.a. & $3.75^{* 5}$ \\
PC nucleus & 6.87 & $8.21^{* 3}$ \\
PC body & $0.99^{* 3}$ & $4.23^{* 3}$ \\
Blood vessel & $4.54^{* 2}$ & $4.54^{* 2}$ \\
\hline Total empty beam & & \\
intensity per projection & $5.85 / 2.14$ & $2.04 / 2.03$ \\
before/after scan $\left(\cdot 10^{10}\right.$, ADU $)$ & & \\
\hline
\end{tabular}
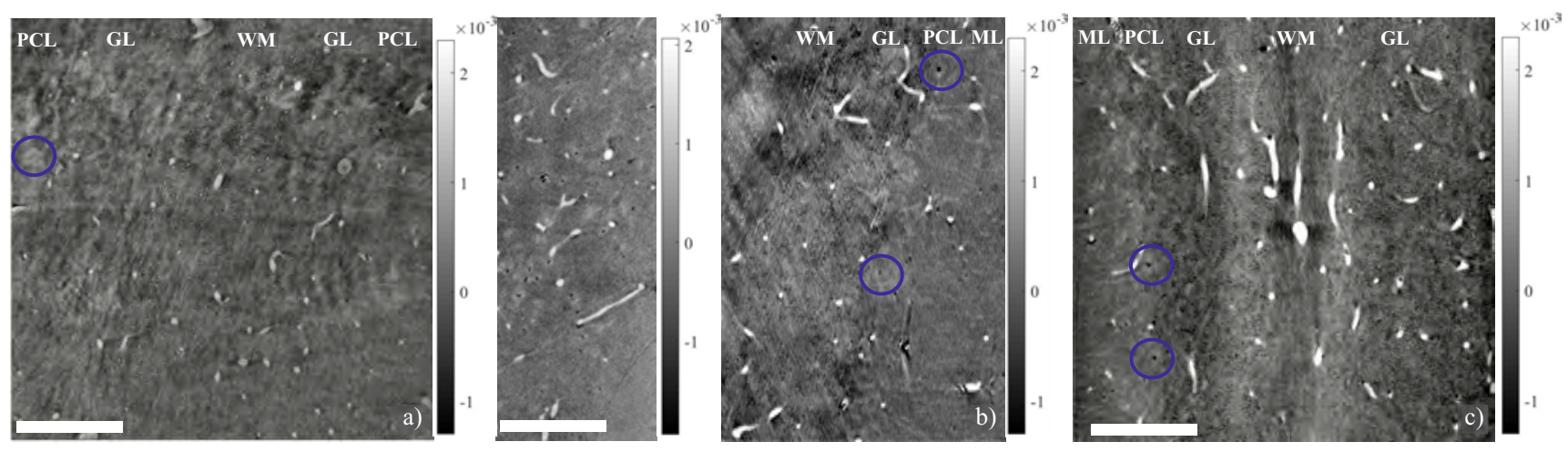

Figure 6. Overview of SR-CT results for the stained samples, given as phase shift per voxel. Stains are (a to c): OsO 4 (30min, "flat"), $\mathrm{OsO}_{4}$ \& PTA (60min, "block" \& "flat", respectively), $\mathrm{OsO}_{4}$ \& UA (60min, "block"). Circles mark PC. Preparation parameters and quality measures are tabulated in Tab. 2. Scale bars: $90 \mu m$. 
Table 2. Metrics for data quality for the stained samples. "b" stands for "block", "f" for "flat". Except for $+(150 \times 150 \times$ $150)$, FSC is based on volumes of $200 \times 200 \times 200$ voxels, with a Kaiser-Bessel window of 7 pixels. ${ }^{66}$ Steepness-of-edge calculated on blood vessels. The SNR was calculated as $\left(\mu_{\mathrm{ft}}-\mu_{\mathrm{bg}}\right) / \sigma_{\mathrm{bg}}$ on neuronal fibers in white matter, Purkinje cells' body and nucleus and blood vessels, respectively. Annotations on SNR marked with * give number of features the number is based on. Empty beam intensities are given in analog-digital-units (ADU).

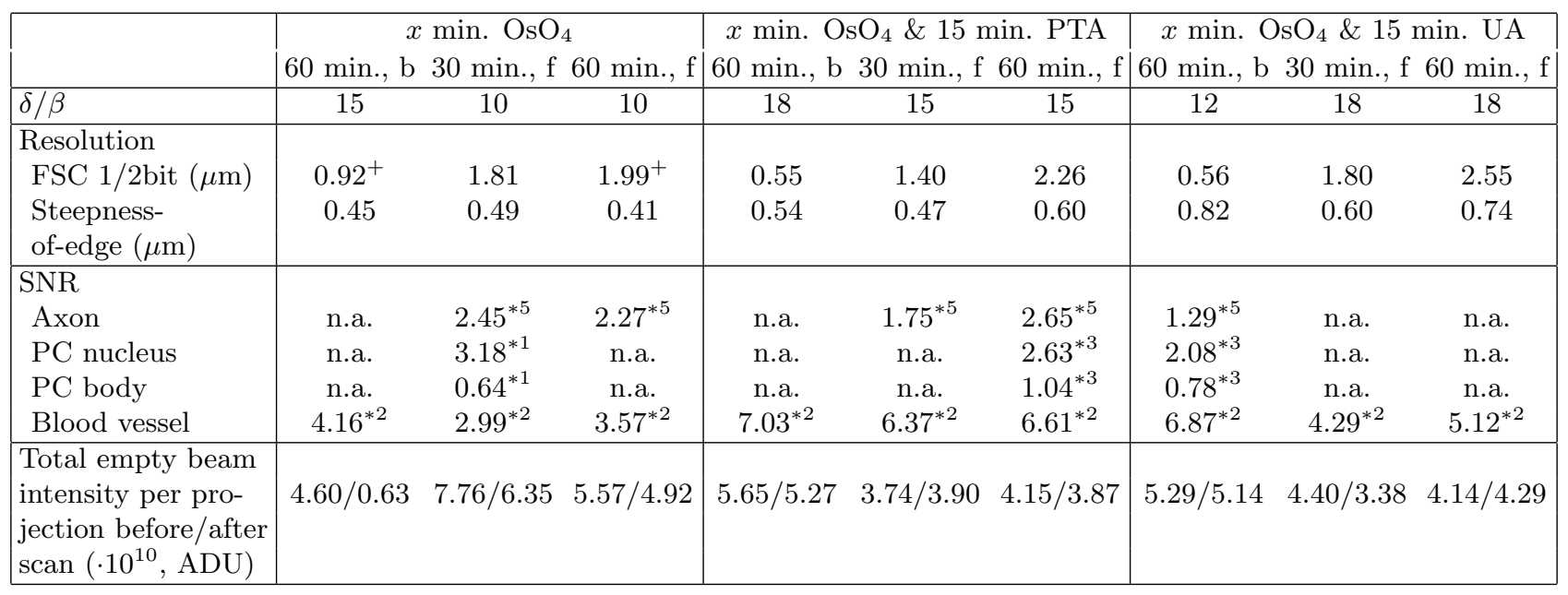

SNR, this sample was scanned also at higher geometric magnification, resulting in $p x_{\text {eff }}=46.8 \mathrm{~nm}$. In addition, the sample was used for segmentation of blood vessels. Fig. 7 shows slices through both reconstructions, the $p x_{\text {eff }}=176 \mathrm{~nm}$ and $p x_{\text {eff }}=46.8 \mathrm{~nm}$ datasets. The high $M$ dataset shows impressive level of detail concerning axons in the FT. It is difficult to appreciate the structure based on one or two slices, but to date we have not yet a segmentation workflow at hand. Instead we illustrate $3 \mathrm{~d}$ segmentation for (the much easier) case of the blood vessels, which appear as a $3 \mathrm{~d}$ network (red) after semi-automatic segmentation, using e.g. Avizo (Thermo Fisher Scientific, Waltham MA, USA). As mentioned above, the negative staining of blood vessels permits a very clear visualization of the vessel network. A first attempt of manual segmentation of fibers is shown in (green).

\subsection{Golgi-Cox-based preparations}

A mouse brain was stained according to the Golgi-Cox protocol described in section 2.2.2. Prior to the extraction of punches, the full mouse brain was imaged with the laboratory setup. BAC-phase retrieval was carried out with $\alpha_{\mathrm{BAC}}=0.1$ and $\gamma_{\mathrm{BAC}}=0.16$. Fig. 8 (a) confirms that the mercury stain penetrated the tissue to full extent. Yet, at the edges of the tissue, low staining contrast emerges, presumingly since the metal has dissolved again. In parallel-beam geometry and at $13.8 \mathrm{keV}$ x-rays at the GINIX instrument, a $1 \mathrm{~mm}$-sample was obtained as described and scanned. $\alpha_{\mathrm{BAC}}=0.002$ and $\gamma_{\mathrm{BAC}}=0.16$ has been used for phase retrieval of this dataset. The semi-automatic rendering of PCs and their dendrites and also the maximum intensity projection demonstrate the particular contrasting of these features. Accordingly, the orientation in a 3d space can be assessed. Further features are contrasted, such as fibers in the GL and WM. At the same time, data also show fractionated staining of features, pointing towards preparation issues. The root of this was examined systematically in further trials. As the impregnation with the solution-D\&E-mixture causes the formation of metallic mercuric sulfides, cerebellum blocks were first cut into $1 \mathrm{~mm}$-slices to then allow for better penetration of the reagent. In addition, the impregnation was prolonged to $1 h$. According to our first laboratory tests, this was found to benefit staining of single neuronal structures. Moreover, increased surrounding temperature to $60^{\circ} \mathrm{C}$ for hours during paraffin embedding may lead to the contrast gradient reported in section 3.2. Keeping the sample hydrated, no such defect was observed in first tests.

\section{DISCUSSION AND OUTLOOK}

In summary, we have presented a first test of different $r O T O$-derived heavy-metal stains for their suitability in $\mathrm{x}$-ray propagation-based phase-contrast tomography. The overall result for contrast of the cytoarchitecture was 

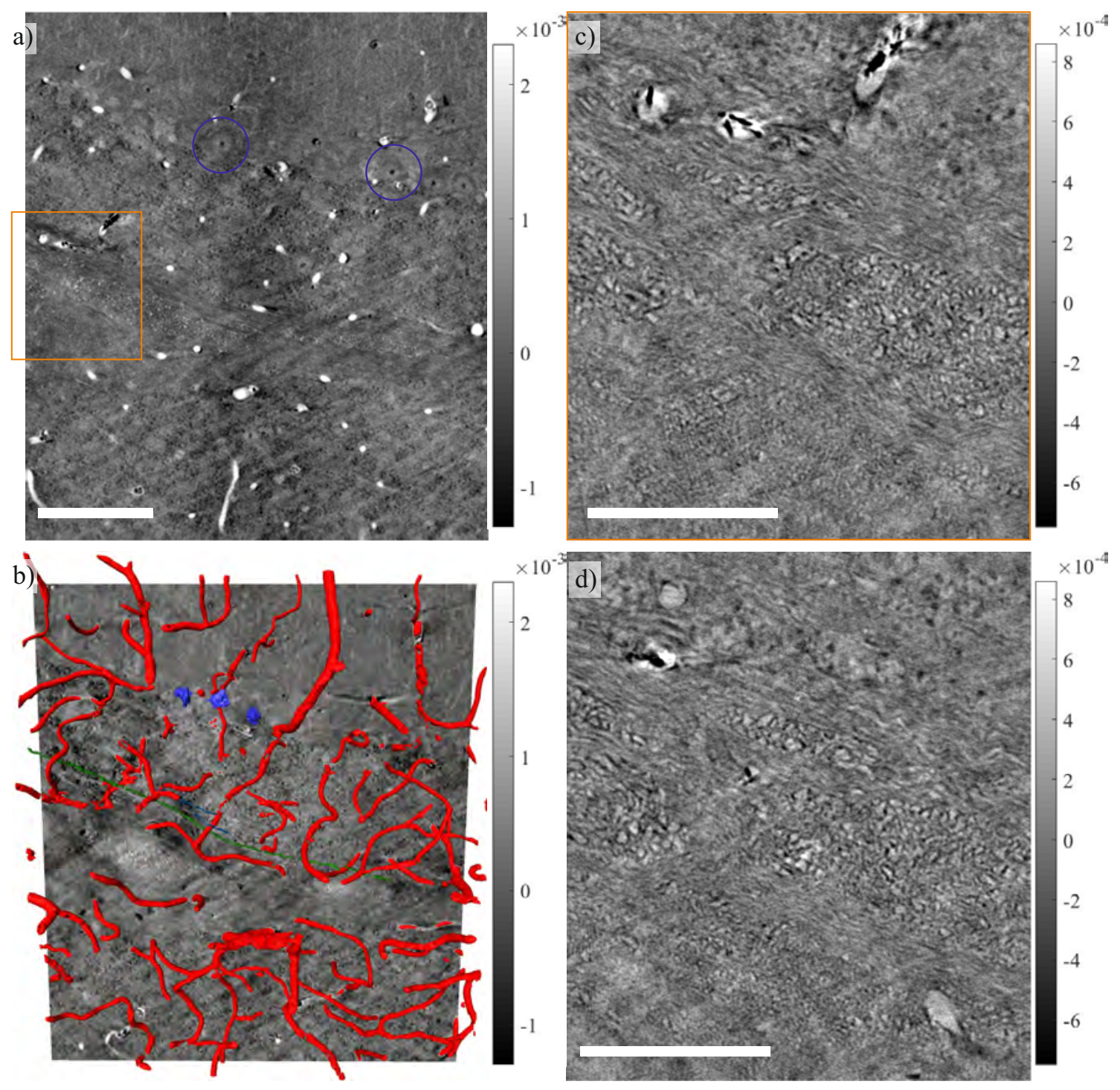

Figure 7. Detailed inspection of the $60 \mathrm{~min} \mathrm{OsO}_{4} \& 15 \mathrm{~min}$ PTA "flat" preparation. (a) virtual slice through the 3d volume of the standard synchrotron scan (as in Fig. 6). The orange square marks the region of the close-up scan shown in (c) and in a different position in (d). Data are presented as phase shift per voxel. (b) Volume rendering of manually segmented features: (red) blood vessels, (violet) PC bodies as also marked in (a), (green) axon fiber. Scale bars: (a \& b) $90 \mu m,(c \&$ d) $40 \mu m$. 

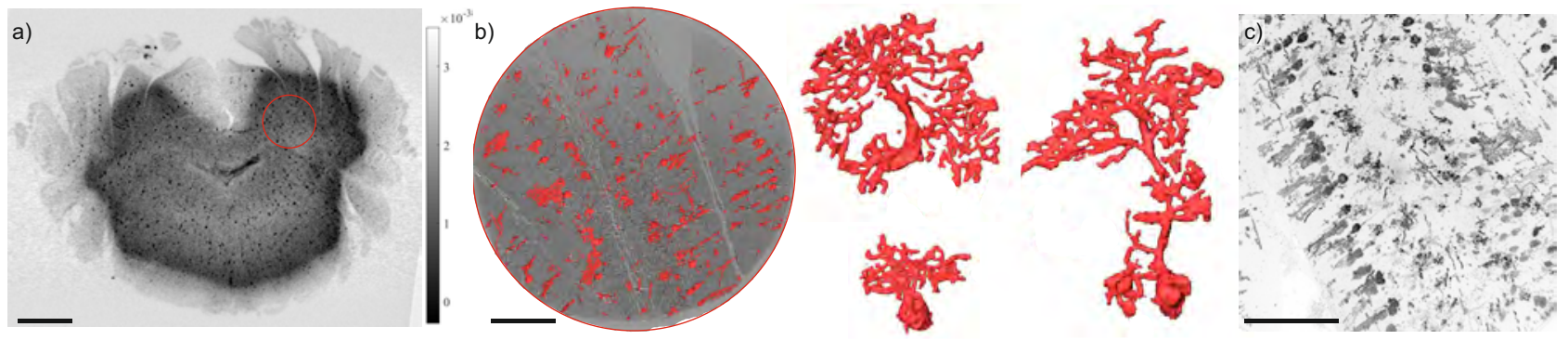

Figure 8. Golgi-Cox-stained mouse cerebellum. (a) Lab- $\mu$ CT result, presenting the effective absorption coefficient per voxel. The red circle indicates the position of the $1 \mathrm{~mm}$-biopsy punch. (b) Volume rendering of the SR-CT result after semi-automatic segmentation of the PCs with the corresponding dendritic trees; the gray slice underneath permits anatomic correlation to the position marked in the overview data. (c) Maximum intensity projection. Scale bars: (a) $1 \mathrm{~mm},(\mathrm{~b} \& \mathrm{c}) 200 \mu \mathrm{m}$.

rather disappointing on the cellular level, while contrasting of larger areas of the cerebellum (e.g. ML versus GL) worked well for large overviews obtained at lab- $\mu \mathrm{CT}$. The situation of heavy-metal stains versus unstained tissue seems to be reciprocal in EM and phase-contrast x-ray microscopy. While the latter gives already excellent results for unstained tissue, as also demonstrated for (high-resolution) SR-CT scans of human cerebellar tissue, ${ }^{5}$ in particular regarding the cytoarchitecture, the use of heavy-metal stains even degrades feature visibility to some extent, whereas most EM techniques are almost useless for unstained tissue. Certainly, this may be explained by current deficits of the protocols as well as not-yet identified small flaws in the preparation. Notwithstanding important further modifications and extensions, we still face an 'overstaining' for x-ray phase contrast. The $\beta / \delta$-ratio may change in a non-optimal way, since presently used stains affect absorption much more than the overall electron density and hence the phase shift. Despite the enhanced absorption, we did not observe motion artifacts due to heating for the present experimental setting as reported in. ${ }^{5}$ Optimizing the $\beta / \delta$-ratio also calls for more rigorous simulations of the optical properties based on stochiometry models and the associated concentration levels.

Preparation details including fixation, embedding and mounting of the samples and the choice of photon energy should be carefully reconsidered, since feature contrasting is not solely based on contrast agents, but can also largely be determined by chemical fixation and tissue embedding media. This also calls for a thorough reinvestigation of the best staining strategy. New approaches like the one in $^{16}$ based on atoms with intermediate $Z$ such as Bromide may point to the right direction. At the same time we must acknowledge that $\mathrm{OsO}_{4}$-based staining has already given excellent results for phase-contrast tomography of the peripheral nervous system $(\mathrm{PNS}),{ }^{4}$ and that PTA and iodine stains has become a gold standard for small animal tomography, see the review of. ${ }^{67}$ Golgi-Cox, also delivers according to expectation, but of course with its characteristic limitations associated with statistical and sparse labeling. For CNS, where contrast for dendrites and synapses in large volume would be a game-changer, we therefore do not yet have a solution at hand. The obvious extension of this work, is to investigate the quality loss/gain situation step-by-step for the protocol and to systematically vary the relevant parameters. While we await such results, we can only recommend phase-contrast imaging of unstained tissues, with proper choice of embedding medium for contrast variation. ${ }^{68}$ To this end, we also stress that the K\&S fixated unstained sample scanned in this work, shows particularly convincing structure preservation, underlining the importance of the fixative.

We do not want to end the report, without a note in passing: as expected, $\mathrm{OsO}_{4}$ does convincingly increases contrast for myelineated neuronal structures in the fibre tracts of cerebellum. As an outlook, we anticipate that this could be propably refined and exploited in future to study the myeloarchitecture, and hence could uniquely contribute to connectomics on larger scales.

\section{ACKNOWLEDGMENTS}

We thank Prof. Dr. Christine Stadelmann and Dr. Franziska van der Meer (Institute for Neuropathology, UMG Göttingen) for human autopsy sample provision. Further, we thank Dr. Michael Sprung and Dr. Fabian 
Westermeier (DESY, Hamburg) for support at P10, Bastian Hartmann for engineering and Susanne Hengst for technical support. We acknowledge financial support by EXC 2067: Multiscale Bioimaging: From Molecular Machines to Networks of Excitable Cells of the Deutsche Forschungsgemeinschaft (DFG).

\section{REFERENCES}

1. Stampanoni, M., Groso, A., Isenegger, A., Mikuljan, G., Chen, Q., Meister, D., Lange, M., Betemps, R., Henein, S., and Abela, R., "TOMCAT: A beamline for TOmographic Microscopy and Coherent rAdiology experimenTs," AIP Conf. Proc. 879, 848-851 (2007).

2. Bech, M., Jensen, T. H., Bunk, O., Donath, T., David, C., Weitkamp, T., Duc, G. L., Bravin, A., Cloetens, P., and Pfeiffer, F., "Advanced contrast modalities for x-ray radiology: Phase-contrast and dark-field imaging using a grating interferometer," J. Med. Phys. 20, 7-16 (2010).

3. Krenkel, M., Töpperwien, M., Bartels, M., Lingor, P., Schild, D., and Salditt, T., "X-ray phase contrast tomography from whole organ down to single cells," Proc. SPIE 9212, 92120R (2014).

4. Bartels, M., Krenkel, M., Cloetens, P., Möbius, W., and Salditt, T., "Myelinated mouse nerves studied by x-ray phase contrast zoom tomography," J. Struct. Biol. 192, 561-568 (2015).

5. Khimchenko, A., Bikis, C., Pacureanu, A., Hieber, S. E., Thalmann, P., Deyhle, H., Schweighauser, G., Hench, J., Frank, S., Müller-Gerbl, M., et al., "Hard X-Ray Nanoholotomography: Large-Scale, Label-Free, 3D Neuroimaging beyond Optical Limit," Adv. Sci. 5, 1700694 (2018).

6. Hieber, S. E., Bikis, C., Khimchenko, A., Schweighauser, G., Hench, J., Chicherova, N., Schulz, G., and Müller, B., "Tomographic brain imaging with nucleolar detail and automatic cell counting," Sci. Rep. 6, 32156 (2016).

7. Töpperwien, M., Van der Meer, F., Stadelmann, C., and Salditt, T., "Three-dimensional virtual histology of human cerebellum by X-ray phase-contrast tomography," PNAS 115(27), 6940-6945 (2018).

8. Müller, B., Fischer, J., Dietz, U., Thurner, P. J., and Beckmann, F., "Blood vessel staining in the myocardium for 3d visualization down to the smallest capillaries," Nucl. Instrum. Methods Phys. Res. Section B: Beam Interactions with Materials and Atoms 246, 254-261 (2006).

9. Metscher, B. D., "Microct for comparative morphology: simple staining methods allow high-contrast 3d imaging of diverse non-mineralized animal tissues," BMC Physiol. 9(1), 11 (2009).

10. Dunmore-Buyze, P. J., Tate, E., li Xiang, F., Detombe, S. A., Nong, Z., Pickering, J. G., and Drangova, M., "Three-dimensional imaging of the mouse heart and vasculature using micro-CT and whole-body perfusion of iodine or phosphotungstic acid," Contrast Media Mol. Imaging 9, 383-390 (2014).

11. Lareida, A., Beckmann, F., Schrott-Fischer, A., Glueckert, R., Freysinger, W., and Müller, B., "Highresolution x-ray tomography of the human inner ear: synchrotron radiation-based study of nerve fibre bundles, membranes and ganglion cells," J. Microsc. 234, 95-102 (2009).

12. Metscher, B. D., "X-ray microtomographic imaging of intact vertebrate embryos," Cold Spring Harb. Protoc. 2011, 1462-1471 (2011).

13. White, D. L., Andrews, S., Faller, J., and Barrnett, R. J., "The chemical nature of osmium tetroxide fixation and staining of membranes by x-ray photoelectron spectroscopy," Biochim. Biophys. Acta - Biomembranes 436, 577-592 (1976).

14. Töpperwien, M., Krenkel, M., Ruhwedel, T., Möbius, W., Pacureanu, A., Cloetens, P., and Salditt, T., "Phase-contrast tomography of sciatic nerves: image quality and experimental parameters," J. Phys: Conf. Ser. 849(1), 012001 (2017).

15. Töpperwien, M., Krenkel, M., Quade, F., and Salditt, T., "Laboratory-based x-ray phase-contrast tomography enables 3D virtual histology," Proc. SPIE 9964, 99640I (2016).

16. Busse, M., Müller, M., Kimm, M. A., Ferstl, S., Allner, S., Achterhold, K., Herzen, J., and Pfeiffer, F., "Three-dimensional virtual histology enabled through cytoplasm-specific X-ray stain for microscopic and nanoscopic computed tomography," PNAS 115(10), 2293-2298 (2018).

17. Helmstaedter, M., Briggman, K. L., Turaga, S. C., Jain, V., Seung, H. S., and Denk, W., "Connectomic reconstruction of the inner plexiform layer in the mouse retina," Nature 500, 168-174 (2013).

18. Helmstaedter, M., "Cellular-resolution connectomics: challenges of dense neural circuit reconstruction," Nat. Meth. 10, 501-507 (2013). 
19. Hua, Y., Laserstein, P., and Helmstaedter, M., "Large-volume en-bloc staining for electron microscopy-based connectomics," Nat. Commun. 6, 7923 (2015).

20. Mikula, S., Binding, J., and Denk, W., "Staining and embedding the whole mouse brain for electron microscopy," Nat. Meth. 9, 1198-1201 (2012).

21. Mikula, S. and Denk, W., "High-resolution whole-brain staining for electron microscopic circuit reconstruction," Nat. Meth. 12(6), 541 (2015).

22. Khimchenko, A., Deyhle, H., Schulz, G., Schweighauser, G., Hench, J., Chicherova, N., Bikis, C., Hieber, S. E., and Müller, B., "Extending two-dimensional histology into the third dimension through conventional micro computed tomography," NeuroImage 139, 26-36 (2016).

23. Schulz, G., Weitkamp, T., Zanette, I., Pfeiffer, F., Beckmann, F., David, C., Rutishauser, S., Reznikova, E., and Müller, B., "High-resolution tomographic imaging of a human cerebellum: comparison of absorption and grating-based phase contrast," J. R. Soc. Interface 7(53), 1665-1676 (2010).

24. Zamir, A., Arthurs, O. J., Hagen, C. K., Diemoz, P. C., Brochard, T., Bravin, A., Sebire, N. J., and Olivo, A., "X-ray phase contrast tomography proof of principle for post-mortem imaging," BJR 89, 20150565 (2016).

25. Saccomano, M., Albers, J., Tromba, G., Radmilović, M. D., Gajović, S., Alves, F., and Dullin, C., "Synchrotron inline phase contrast $\mu \mathrm{CT}$ enables detailed virtual histology of embedded soft-tissue samples with and without staining," J. Synchrotron Radiat. 25, 1153-1161 (2018).

26. Willingham, M. C. and Rutherford, A. V., "The use of osmium-thiocarbohydrazide-osmium (OTO) and ferrocyanide-reduced osmium methods to enhance membrane contrast and preservation in cultured cells.," J. Histochem. Cytochem. 32, 455-460 (1984).

27. Hayat, M. A., [Principles and techniques of electron microscopy. Biological applications, volume 1.] (1974).

28. Robinson, D. G., Ehlers, U., Herken, R., Herrmann, B., Mayer, F., and Schürmann, F.-W., [Methods of Preparation for Electron Microscopy], Springer Berlin Heidelberg (1987).

29. de Castro Fonseca, M., Araujo, B. H. S., Dias, C. S. B., Archilha, N. L., Neto, D. P. A., Cavalheiro, E., Westfahl, H., da Silva, A. J. R., and Franchini, K. G., "High-resolution synchrotron-based x-ray microtomography as a tool to unveil the three-dimensional neuronal architecture of the brain," Sci. Rep. 8, 12074 (2018).

30. Töpperwien, M., Krenkel, M., Müller, K., and Salditt, T., "Phase-contrast tomography of neuronal tissues: from laboratory-to high resolution synchrotron CT," Proc. SPIE 9967, 99670T (2016).

31. Das, G., Reuhl, K., and Zhou, R., "The golgi-cox method," in [Methods in Molecular Biology], 313-321, Humana Press (2013).

32. Golgi, C., "Sulla struttura della sostanza grigia del cervello," Gazz. Med. Ital. - Lombardia 6, 244-246 (1873).

33. Golgi, C., "Di una nuova reasione apparentemente nera delle cellule nervose cerebrali ottenuta col bichloruro di mercurio," Arch. Med. Sci. 3, 1-7 (1879).

34. Golgi, C., "Modificazione del metodo di colorazione deli elementi nervosi col bichloruru di mercurio," La Rivista Italiana della Medicina di Laboratorio 7, 193-194 (1891).

35. Cajal, S., "¿neuronismo o reticularismo? las pruebas objetivas de la unidad anatómica de las células nerviosas," Arch. Neurobiol. 13, 1-44 (1933).

36. Roberts, W., Campbell, T., and Rapp, G., [Encyclopedia of Minerals], Van Nostrand Reinhold, 2 ed. (1990).

37. Bartels, M., Hernandez, V. H., Krenkel, M., Moser, T., and Salditt, T., "Phase contrast tomography of the mouse cochlea at microfocus x-ray sources," Appl. Phys. Lett. 103(8), 083703 (2013).

38. Töpperwien, M., Krenkel, M., Vincenz, D., Stöber, F., Oelschlegel, A. M., Goldschmidt, J., and Salditt, T., "Three-dimensional mouse brain cytoarchitecture revealed by laboratory-based x-ray phase-contrast tomography," Sci. Rep. 7, 42847 (2017).

39. Salditt, T., Osterhoff, M., Krenkel, M., Wilke, R. N., Priebe, M., Bartels, M., Kalbfleisch, S., and Sprung, M., "Compound focusing mirror and X-ray waveguide optics for coherent imaging and nano-diffraction," J. Synchrotron Radiat. 22, 867-878 (2015).

40. Paganin, D. M., [Coherent X-Ray Optics], New York: Oxford University (2006). 
41. Witte, Y. D., Boone, M., Vlassenbroeck, J., Dierick, M., and Hoorebeke, L. V., "Bronnikov-aided correction for x-ray computed tomography," J. Opt. Soc. Am. A 26, 890-894 (2009).

42. Groso, A., Abela, R., and Stampanoni, M., "Implementation of a fast method for high resolution phase contrast tomography," Opt. Express 14, 8103-8110 (2006).

43. Groso, A., Stampanoni, M., Abela, R., Schneider, P., Linga, S., and Müller, R., "Phase contrast tomography: An alternative approach," Appl. Phys. Lett. 88, 214104 (2006).

44. Cloetens, P., Ludwig, W., Baruchel, J., Van Dyck, D., Van Landuyt, J., Guigay, J. P., and Schlenker, M., "Holotomography: Quantitative phase tomography with micrometer resolution using hard synchrotron radiation x rays," Appl. Phys. Lett. 75(19), 2912-2914 (1999).

45. Guigay, J. P., "Fourier transform analysis of fresnel diffraction patterns and in-line holograms," Optik 49(1), 121-125 (1977).

46. Zabler, S., Cloetens, P., Guigay, J.-P., Baruchel, J., and Schlenker, M., "Optimization of phase contrast imaging using hard x rays," Rev. Sci. Instrum. 76(7), 073705 (2005).

47. Krenkel, M., Toepperwien, M., Alves, F., and Salditt, T., "Three-dimensional single-cell imaging with X-ray waveguides in the holographic regime," Acta Crystallogr. A 73, 282-292 (2017).

48. Krenkel, M., Töpperwien, M., Dullin, C., Alves, F., and Salditt, T., "Propagation-based phase-contrast tomography for high-resolution lung imaging with laboratory sources," AIP Advances 6(3), 035007 (2016).

49. Tuohimaa, T., Otendal, M., and Hertz, H. M., "Phase-contrast x-ray imaging with a liquid-metal-jet-anode microfocus source," Appl. Phys. Lett. 91(7), 074104 (2007).

50. Vågberg, W., Larsson, D. H., Li, M., Arner, A., and Hertz, H. M., "X-ray phase-contrast tomography for high-spatial-resolution zebrafish muscle imaging," Sci. Rep. 5(16625), 1-6 (2015).

51. Larsson, D. H., Vågberg, W., Yaroshenko, A., Yildirim, A. Ö., and Hertz, H. M., "High-resolution shortexposure small-animal laboratory x-ray phase-contrast tomography," Sci. Rep. 6, 39074 (2016).

52. Vågberg, W., Persson, J., Szekely, L., and Hertz, H. M., "Cellular-resolution 3D virtual histology of human coronary arteries using x-ray phase tomography," Sci. Rep. 8(1), 11014 (2018).

53. Töpperwien, M., Doeppner, T. R., Zechmeister, B., Bähr, M., and Salditt, T., "Multiscale x-ray phasecontrast tomography in a mouse model of transient focal cerebral ischemia," Biomed. Opt. Express 10, 92-103 (2019).

54. Palenstijn, W. J., Batenburg, K. J., and Sijbers, J., "Performance improvements for iterative electron tomography reconstruction using graphics processing units (GPUs)," J. Struct. Biol. 176(2), 250-253 (2011).

55. van Aarle, W., Palenstijn, W. J., De Beenhouwer, J., Altantzis, T., Bals, S., Batenburg, K. J., and Sijbers, J., "The ASTRA Toolbox: A platform for advanced algorithm development in electron tomography," Ultramicroscopy 157, 35-47 (2015).

56. Münch, B., Trtik, P., Marone, F., and Stampanoni, M., "Stripe and ring artifact removal with combined wavelet-fourier filtering," Opt. Express 17(10), 8567-8591 (2009).

57. Osterhoff, M. and Salditt, T., "Coherence filtering of x-ray waveguides: analytical and numerical approach," New J. Phys. 13(10), 103026 (2011).

58. Krüger, S. P., Giewekemeyer, K., Kalbfleisch, S., Bartels, M., Neubauer, H., and Salditt, T., "Sub-15 nm beam confinement by twocrossed x-ray waveguides," Opt. Express 18, 13492-13501 (2010).

59. Krüger, S. P., Neubauer, H., Bartels, M., Kalbfleisch, S., Giewekemeyer, K., Wilbrandt, P. J., Sprung, M., and Salditt, T., "Sub-10 nm beam confinement by X-ray waveguides: design, fabrication and characterization of optical properties," J. Synchrotron Radiat. 19(2), 227-236 (2012).

60. Bartels, M., Krenkel, M., Haber, J., Wilke, R. N., and Salditt, T., "X-ray holographic imaging of hydrated biological cells in solution," Phys. Rev. Lett. 114, 048103 (2015).

61. Neubauer, H., Hoffmann, S., Kanbach, M., Haber, J., Kalbfleisch, S., Krüger, S. P., and Salditt, T., "High aspect ratio x-ray waveguide channels fabricated by e-beam lithography and wafer bonding," J. Appl. Phys. 115(21), 214305 (2014).

62. Ketcham, R. A., "New algorithms for ring artifact removal," Proc. SPIE 6318, $63180 \mathrm{O}$ (2006).

63. Weil, M.-T., Heibeck, S., Töpperwien, M., tom Dieck, S., Ruhwedel, T., Salditt, T., Rodicio, M. C., Morgan, J. R., Nave, K.-A., Möbius, W., and Werner, H. B., "Axonal ensheathment in the nervous system of lamprey: Implications for the evolution of myelinating glia," Journal of Neuroscience 38(29), 6586-6596 (2018). 
64. Ramón-Moliner, E., "The golgi-cox technique," in [Contemporary Research Methods in Neuroanatomy], 32-55, Springer Berlin Heidelberg (1970).

65. Glaser, E. M. and der Loos, H. V., "Analysis of thick brain sections by obverse-reverse computer microscopy: Application of a new, high clarity golgi-nissl stain," J. Neurosci. Methods 4, 117-125 (1981).

66. Butz, T., [Fouriertransformation für Fußgänger], Vieweg+Teubner Verlag / Springer Fachmedien Wiesbaden GmbH, Wiesbaden (2012).

67. Gutiérrez, Y., Ott, D., Töpperwien, M., Salditt, T., and Scherber, C., "X-ray computed tomography and its potential in ecological research: A review of studies and optimization of specimen preparation," Ecol. Evol. 8(15), 7717-7732 (2018).

68. Töpperwien, M., Markus, A., Alves, F., and Salditt, T., "Contrast enhancement for visualizing neuronal cytoarchitecture by propagation-based x-ray phase-contrast tomography," NeuroImage 199, 70-80 (2019). 